Proc. Indian Acad. Sci. (Math. Sci.) Vol. 114, No. 2, May 2003, pp. 203-213.

Printed in India

\title{
Unsteady Stokes equations: Some complete general solutions
}

\author{
A VENKATLAXMI, B S PADMAVATHI and T AMARANATH \\ Department of Mathematics and Statistics, University of Hyderabad, \\ Hyderabad 500 046, India \\ E-mail: tasm@uohyd.ernet.in
}

MS received 2 December 2003

\begin{abstract}
The completeness of solutions of homogeneous as well as nonhomogeneous unsteady Stokes equations are examined. A necessary and sufficient condition for a divergence-free vector to represent the velocity field of a possible unsteady Stokes flow in the absence of body forces is derived.
\end{abstract}

Keywords. Complete general solution; unsteady Stokes flow.

\section{Unsteady Stokes flows: Homogeneous case}

The equations governing the motion of an arbitrary unsteady Stokes flow of an incompressible, viscous fluid in the absence of any body forces are

$$
\begin{aligned}
\rho \frac{\partial \mathbf{V}}{\partial t} & =-\nabla p+\mu \nabla^{2} \mathbf{V} \\
\nabla \cdot \mathbf{V} & =0
\end{aligned}
$$

where $\mathbf{V}$ is the velocity, $p$ is the pressure, $\rho$ is the density and $\mu$ is the coefficient of dynamic viscosity of the fluid. Equation (1) can also be written as

$$
\mu\left(\nabla^{2}-\frac{1}{v} \frac{\partial}{\partial t}\right) \mathbf{V}=\nabla p
$$

where $v=(\mu / \rho)$ is the kinematic coefficient of viscosity. Taking divergence of eq. (3) and making use of eq. (2), it is easy to see that the pressure is harmonic. Hence, on operating the Laplace operator on eq. (3), we find that the velocity vector satisfies the equation

$$
\nabla^{2}\left(\nabla^{2}-\frac{1}{v} \frac{\partial}{\partial t}\right) \mathbf{V}=0
$$

\subsection{A complete general solution of unsteady Stokes equations}

Let $(\mathbf{V}, p)$ be any solution of (2) and (3). We define

$$
\psi=\int_{0}^{t} \mathrm{~d} \tau \iiint_{D} p(\xi, \eta, \zeta, \tau)\left(\frac{\exp \left[-r^{2} /(4 v(t-\tau))\right]}{v^{1 / 2}[4(t-\tau)]^{3 / 2}}\right) \mathrm{d} \xi \mathrm{d} \eta \mathrm{d} \zeta
$$


where $D$ is the region of the flow, which is simply connected and

$$
r=\left[(x-\xi)^{2}+(y-\eta)^{2}+(z-\zeta)^{2}\right]^{1 / 2} .
$$

Then

$$
\left(\nabla^{2}-\frac{1}{v} \frac{\partial}{\partial t}\right) \psi=p(x, y, z, t)
$$

Hence

$$
\nabla^{2}\left(\nabla^{2}-\frac{1}{v} \frac{\partial}{\partial t}\right) \psi=0
$$

Substituting (6) in (3), we get

$$
\left(\nabla^{2}-\frac{1}{v} \frac{\partial}{\partial t}\right)\left[\mathbf{V}-\frac{1}{\mu} \nabla \psi\right]=0
$$

Hence

$$
\mathbf{V}=\Phi+\frac{1}{\mu} \nabla \psi
$$

where

$$
\left(\nabla^{2}-\frac{1}{v} \frac{\partial}{\partial t}\right) \Phi=0
$$

Substituting the expression (9) in eq. (2), we get

$$
\nabla^{2} \psi=-\mu \nabla \cdot \Phi
$$

A general solution of eq. (7) is of the form $\psi=\psi_{1}+\psi_{2}$, where

$$
\begin{aligned}
& \nabla^{2} \psi_{1}=0, \\
& \left(\nabla^{2}-\frac{1}{v} \frac{\partial}{\partial t}\right) \psi_{2}=0,
\end{aligned}
$$

(please see appendix).

Hence from eq. (6), we have

$$
\begin{aligned}
p & =\left(\nabla^{2}-\frac{1}{v} \frac{\partial}{\partial t}\right)\left(\psi_{1}+\psi_{2}\right) \\
& =-\frac{1}{v} \frac{\partial \psi_{1}}{\partial t} .
\end{aligned}
$$

Using eqs (11) and (12), we get

$$
\nabla^{2} \psi_{2}=-\mu \nabla \cdot \Phi,
$$

which implies that

$$
\frac{1}{v} \frac{\partial \psi_{2}}{\partial t}=-\mu \nabla \cdot \Phi
$$


Therefore

$$
\frac{\partial \psi_{2}}{\partial t}=-\mu \nu \nabla \cdot \Phi \quad \text { or } \quad \psi_{2}=-\int_{0}^{t} \mu \nu \nabla \cdot \Phi(x, y, z, s) \mathrm{d} s
$$

as $\psi_{2}(x, y, z, 0)=0$.

So

$$
\begin{aligned}
\mathbf{V} & =\Phi+\frac{1}{\mu} \nabla \psi \\
& =\Phi+\frac{1}{\mu} \nabla \psi_{1}+\frac{1}{\mu} \nabla \psi_{2} \\
& =\Phi+\frac{1}{\mu} \nabla \psi_{1}-v \nabla \int_{0}^{t} \nabla \cdot \Phi(x, y, z, s) \mathrm{d} s .
\end{aligned}
$$

This solution is similar to the Naghdi-Hsu type of solution [3] given for steady Stokes equations.

\section{Non-homogeneous case}

Consider the equations of motion of an unsteady Stokes flow of a viscous, incompressible fluid in the presence of a body force $\mathbf{f}$ given by

$$
\begin{aligned}
\rho \frac{\partial \mathbf{V}}{\partial t} & =-\nabla p+\mu \nabla^{2} \mathbf{V}+\mathbf{f}, \\
\nabla \cdot \mathbf{V} & =0 .
\end{aligned}
$$

The function $\mathbf{f}$ can be represented as [2]

$$
\mathbf{f}=\nabla \chi+\nabla \times \nabla \times(\mathbf{r} P)+\nabla \times(\mathbf{r} T),
$$

where $\chi, P$ and $T$ are the given scalar functions. Taking divergence of eq. (19) and making use of eqs (20) and (21), we observe that

$$
\nabla^{2}(\chi-p)=0 \text {. }
$$

Hence

$$
p=p^{\prime}+\chi, \text { where } \nabla^{2} p^{\prime}=0 .
$$

Let $S$ be defined as the region

$$
S=\left\{(r, \theta, \varphi): r_{1} \leq r \leq r_{2}, 0 \leq \theta \leq \pi, 0 \leq \varphi \leq 2 \pi\right\} .
$$

Chadwick and Trowbridge [1] have shown that if $\mathbf{V}$ is a vector field possessing partial derivatives of orders up to two which are Hölder continuous on $S$ and $\nabla \cdot \mathbf{V}=0$ on $S$, then there are scalar functions $A$ and $B$ on $S$ such that

$$
\mathbf{V}=\nabla \times \nabla \times(\mathbf{r} A)+\nabla \times(\mathbf{r} B),
$$

where $A$ and $B$ are solutions of

$$
\begin{aligned}
& L A=-\mathbf{r} \cdot \mathbf{V}, \\
& L B=-\mathbf{r} \cdot(\nabla \times \mathbf{V}),
\end{aligned}
$$

where $L$ is the transverse part of the Laplace operator except for the factor $1 / r^{2}$. 
Theorem. A complete general solution of eqs (19) and (20) in S is given by

$$
\begin{aligned}
& \mathbf{V}=\nabla \times \nabla \times(\mathbf{r} A)+\nabla \times(\mathbf{r} B) \\
& p=p_{0}+\chi+\frac{\partial}{\partial r}\left\{r\left[P+\mu\left(\nabla^{2}-\frac{1}{v} \frac{\partial}{\partial t}\right) A\right]\right\}
\end{aligned}
$$

where $A$ and $B$ satisfy equations

$$
\begin{aligned}
& \mu \nabla^{2}\left(\nabla^{2}-\frac{1}{v} \frac{\partial}{\partial t}\right) A+\nabla^{2} P=0, \\
& \mu\left(\nabla^{2}-\frac{1}{v} \frac{\partial}{\partial t}\right) B+T=0,
\end{aligned}
$$

respectively.

Proof. Let $A$ be a solution of eq. (25). Operating $\nabla^{2}\left(\nabla^{2}-\frac{1}{v} \frac{\partial}{\partial t}\right)$ on both sides of (25) and making use of eq. (20), we find that

$$
\begin{aligned}
\nabla^{2}\left(\nabla^{2}-\frac{1}{v} \frac{\partial}{\partial t}\right) L A & =-\nabla^{2}\left(\nabla^{2}-\frac{1}{v} \frac{\partial}{\partial t}\right)(\mathbf{r} \cdot \mathbf{V}) \\
& =-\mathbf{r} \cdot \nabla^{2}\left(\nabla^{2}-\frac{1}{v} \frac{\partial}{\partial t}\right) \mathbf{V}
\end{aligned}
$$

Here we used the identity that

$$
\nabla^{2}(\mathbf{r} \cdot \mathbf{V})=2 \nabla \cdot \mathbf{V}+\mathbf{r} \cdot \nabla^{2} \mathbf{V}
$$

Since the operators $\nabla^{2}, L$ and $\partial / \partial t$ commute, we rewrite eq. (30) as

$$
L \nabla^{2}\left(\nabla^{2}-\frac{1}{v} \frac{\partial}{\partial t}\right) A=-\mathbf{r} \cdot \nabla^{2}\left(\nabla^{2}-\frac{1}{v} \frac{\partial}{\partial t}\right) \mathbf{V} .
$$

We rewrite $\mathbf{f}$ as

$$
\mathbf{f}=\nabla \chi+\nabla\left(P+r \frac{\partial P}{\partial r}\right)-\mathbf{r} \nabla^{2} P+\nabla \times(\mathbf{r} T) .
$$

Taking $\nabla^{2}$ of eq. (19) and making use of (22) and (32), we get

$$
\begin{aligned}
\mu \nabla^{2}\left(\nabla^{2}-\frac{1}{v} \frac{\partial}{\partial t}\right) \mathbf{V} & =\nabla^{2}(\nabla p)-\nabla^{2} \mathbf{f} \\
& =-\nabla^{2} \nabla\left(P+r \frac{\partial P}{\partial r}\right)+\nabla^{2}\left(\mathbf{r} \nabla^{2} P\right)-\nabla^{2}(\nabla \times(\mathbf{r} T)) \\
& =-\nabla\left[\frac{\partial}{\partial r}\left(r \nabla^{2} P\right)\right]+\mathbf{r} \nabla^{4} P-\nabla \times\left(\mathbf{r} \nabla^{2} T\right) .
\end{aligned}
$$

Here, from eqs (31) and (33), we have

$$
\mu L \nabla^{2}\left(\nabla^{2}-\frac{1}{v} \frac{\partial}{\partial t}\right) A=-L \nabla^{2} P
$$


or

$$
L\left[\mu \nabla^{2}\left(\nabla^{2}-\frac{1}{v} \frac{\partial}{\partial t}\right) A+\nabla^{2} P\right]=0 .
$$

From [1], this implies that $\mu \nabla^{2}\left(\nabla^{2}-\frac{1}{v} \frac{\partial}{\partial t}\right) A+\nabla^{2} P=f(r)$, where $f$ is an arbitrary function of $r$. Without loss of generality, $f(r)$ can be neglected as in the proof given in [5]. Then we observe that $A$ satisfies eq. (28).

Similarly, by considering eq. (26), we find that

$$
\mu L\left(\nabla^{2}-\frac{1}{v} \frac{\partial}{\partial t}\right) B=-L T,
$$

or

$$
L\left[\mu\left(\nabla^{2}-\frac{1}{v} \frac{\partial}{\partial t}\right) B+T\right]=0
$$

Following the result given in [1], it follows that

$$
\left[\mu\left(\nabla^{2}-\frac{1}{v} \frac{\partial}{\partial t}\right) B+T\right]=g(r)
$$

where $g$ is an arbitrary function of $r$. Without loss of generality, $g(r)$ can also be neglected as before. Then we find that $B$ satisfies eq. (29).

Substituting (21) and (24) in eq. (19), we find that

$$
\begin{gathered}
\nabla\left[\chi-p+\frac{\partial}{\partial r}(r P)+\mu \frac{\partial}{\partial r}\left(r \nabla^{2} A\right)-\rho \frac{\partial}{\partial r}\left(r A_{t}\right)\right]-\mathbf{r}\left[\nabla^{2} P\right. \\
\left.+\mu \nabla^{4} A-\rho \nabla^{2} A_{t}\right]+\nabla \times\left[\mathbf{r}\left(T+\mu \nabla^{2} B-\rho B_{t}\right)\right]=0
\end{gathered}
$$

Since $A$ and $B$ satisfy eqs (28) and (29), we have

$$
p=p_{0}+\chi+\frac{\partial}{\partial r}\left\{r\left[P+\mu\left(\nabla^{2}-\frac{1}{v} \frac{\partial}{\partial t}\right) A\right]\right\},
$$

where $p_{0}$ is a constant. Hence a complete general solution of eqs (19) and (20) is given by $\mathbf{V}$ and $p$ as given in (24) and (27) respectively, where $A$ and $B$ satisfy eqs (28) and (29) respectively.

\subsection{Alternative proof of completeness}

Let $(\mathbf{V}, p)$ be a solution of (19) and (20). Since $p-\chi$ is harmonic, it is possible to find a harmonic function $\phi$ where

$$
\phi+r \frac{\partial \phi}{\partial r}=p-\chi
$$

and a function $A_{p}$ such that

$$
\mu\left(\nabla^{2}-\frac{1}{v} \frac{\partial}{\partial t}\right) A_{p}=\phi-P
$$


Such a function $A_{p}$ satisfies

$$
\mu \nabla^{2}\left(\nabla^{2}-\frac{1}{v} \frac{\partial}{\partial t}\right) A_{p}=-\nabla^{2} P
$$

We observe that

$$
\begin{aligned}
\mathbf{V}_{1} & =\nabla \times \nabla \times\left(\mathbf{r} A_{p}\right), \\
p & =\chi+\frac{\partial}{\partial r}\left\{r\left[\mu\left(\nabla^{2}-\frac{1}{v} \frac{\partial}{\partial t}\right) A_{p}+P\right]\right\},
\end{aligned}
$$

is a particular solution of (19) and (20), where $\mathbf{f}=\nabla \times \nabla \times(\mathbf{r} P)+\nabla \chi$. Hence every $p$ satisfying $\nabla^{2} p-\nabla^{2} \chi=0$ represents pressure in (19) and (20) for which a possible velocity field is given by (40). Therefore in the homogeneous case, every harmonic $p$ represents the pressure of a possible Stokes flow. Consider

$$
\mathbf{V}_{2}=\mathbf{V}-\mathbf{V}_{1}
$$

where $\mathbf{V}_{1}$ is as given in (40). Then

$$
\begin{aligned}
\left(\nabla^{2}-\frac{1}{v} \frac{\partial}{\partial t}\right) \mathbf{V}_{2} & =\left(\nabla^{2}-\frac{1}{v} \frac{\partial}{\partial t}\right) \mathbf{V}-\left(\nabla^{2}-\frac{1}{v} \frac{\partial}{\partial t}\right) \mathbf{V}_{1} \\
& =0
\end{aligned}
$$

as $\mathbf{V}$ and $\mathbf{V}_{1}$ are both solutions of (19). Since

$$
\nabla \cdot \mathbf{V}_{2}=0
$$

we can write

$$
\mathbf{V}_{2}=\nabla \times \nabla \times\left(\mathbf{r} A_{c}\right)+\nabla \times(\mathbf{r} B),
$$

where

$$
\begin{aligned}
& L A_{c}=-\mathbf{r} \cdot \mathbf{V}_{2}, \\
& L B=-\mathbf{r} \cdot\left(\nabla \times \mathbf{V}_{2}\right) .
\end{aligned}
$$

Then, from (43) and (46)

$$
\left(\nabla^{2}-\frac{1}{v} \frac{\partial}{\partial t}\right) L A_{c}=-\mathbf{r} \cdot\left(\nabla^{2}-\frac{1}{v} \frac{\partial}{\partial t}\right) \mathbf{V}_{2}=0
$$

or

$$
L\left(\nabla^{2}-\frac{1}{v} \frac{\partial}{\partial t}\right) A_{c}=0
$$

Similarly

$$
\begin{aligned}
\mu L\left(\nabla^{2}-\frac{1}{v} \frac{\partial}{\partial t}\right) B & =\mu\left(\nabla^{2}-\frac{1}{v} \frac{\partial}{\partial t}\right) L B \\
& =-\mu\left(\nabla^{2}-\frac{1}{v} \frac{\partial}{\partial t}\right)\left(\mathbf{r} \cdot\left(\nabla \times \mathbf{V}_{2}\right)\right) \\
& =-L T .
\end{aligned}
$$


Hence

$$
\left(\nabla^{2}-\frac{1}{v} \frac{\partial}{\partial t}\right) A_{c}=0
$$

and

$$
\mu\left(\nabla^{2}-\frac{1}{v} \frac{\partial}{\partial t}\right) B+T=0
$$

If we write

$$
\begin{aligned}
& A=A_{p}+A_{c}, \\
& \mu \nabla^{2}\left(\nabla^{2}-\frac{1}{v} \frac{\partial}{\partial t}\right) A=\mu \nabla^{2}\left(\nabla^{2}-\frac{1}{v} \frac{\partial}{\partial t}\right)\left[A_{p}+A_{c}\right]=-\nabla^{2} P,
\end{aligned}
$$

or

$$
\mu \nabla^{2}\left(\nabla^{2}-\frac{1}{v} \frac{\partial}{\partial t}\right) A+\nabla^{2} P=0
$$

Hence

$$
\begin{aligned}
\mathbf{V} & =\mathbf{V}_{1}+\mathbf{V}_{2} \\
& =\nabla \times \nabla \times\left(\mathbf{r} A_{p}\right)+\nabla \times \nabla \times\left(\mathbf{r} A_{c}\right)+\nabla \times(\mathbf{r} B) \\
& =\nabla \times \nabla \times(\mathbf{r} A)+\nabla \times(\mathbf{r} B)
\end{aligned}
$$

and

$$
p=p_{0}+\chi+\frac{\partial}{\partial r}\left\{r\left[P+\mu\left(\nabla^{2}-\frac{1}{v} \frac{\partial}{\partial t}\right) A\right]\right\}
$$

where

$$
\begin{aligned}
& \mu \nabla^{2}\left(\nabla^{2}-\frac{1}{v} \frac{\partial}{\partial t}\right) A+\nabla^{2} P=0, \\
& \mu\left(\nabla^{2}-\frac{1}{v} \frac{\partial}{\partial t}\right) B+T=0 .
\end{aligned}
$$

\section{Condition for a possible Stokes flow}

We now derive a necessary and sufficient condition for a divergence free $\mathbf{V}$ satisfying $\nabla^{2}\left(\nabla^{2}-\frac{1}{v} \frac{\partial}{\partial t}\right) \mathbf{V}=0$, to be the solution of homogeneous, unsteady Stokes equations (1) and (2). We make the following observations:

1. On operating curl on either sides of eq. (3), we find that the vorticity $\nabla \times \mathbf{V}$ satisfies the equation

$$
\left(\nabla^{2}-\frac{1}{v} \frac{\partial}{\partial t}\right)(\nabla \times \mathbf{V})=0
$$

2. If $\left(\mathbf{V}_{1}, p\right)$ and $\left(\mathbf{V}_{2}, p\right)$ are solutions of the homogeneous, unsteady Stokes equations (1) and (2), then $\mathbf{U}=\mathbf{V}_{1}-\mathbf{V}_{2}$ satisfies the equation 


$$
\left(\nabla^{2}-\frac{1}{v} \frac{\partial}{\partial t}\right) \mathbf{U}=0
$$

and $\left(\mathbf{U}, p_{0}\right)$ is also a solution, where $p_{0}$ is a constant.

3. If $\left(\mathbf{V}, p_{1}\right)$ and $\left(\mathbf{V}, p_{2}\right)$ are solutions of the homogeneous, unsteady Stokes equations (1) and (2), then $\left(p_{1}-p_{2}\right)$ is constant.

4. If $(\mathbf{V}, p)$ is a solution of (1) and (2) then $(\nabla \times \mathbf{V}, 0)$ is also a solution.

We now show that every divergence-free $\mathbf{V}$ satisfying

$$
\nabla^{2}\left(\nabla^{2}-\frac{1}{v} \frac{\partial}{\partial t}\right) \mathbf{V}=0
$$

need not be a solution of the homogeneous unsteady Stokes equations (1) and (2). Let us consider

$$
\mathbf{V}=(y \hat{i}-x \hat{j}) \exp (v t),
$$

where $\hat{i}$ and $\hat{j}$ are the unit vectors in the cartesian coordinates $(x, y)$. It can be easily verified that $\nabla \cdot \mathbf{V}=0$ and $\mathbf{V}$ satisfies eq. (4).

Substituting $\mathbf{V}$ in eq. (3) and expressing the equations in a component form we get

$$
\begin{aligned}
& p_{x}=-\mu y \exp (v t), \\
& p_{y}=\mu x \exp (v t) .
\end{aligned}
$$

It can be seen that $p_{x y} \neq p_{y x}$. Therefore it is not possible to find the pressure $p$ corresponding to the velocity $\mathbf{V}$ given in (55) and hence $\mathbf{V}$ is not a solution of the homogeneous, unsteady Stokes equations. We now derive the necessary and sufficient condition for a divergence-free vector $\mathbf{V}$ satisfying eq. (4) to be a possible velocity field in an unsteady Stokes flow in the absence of any body forces.

Theorem. Let $\mathbf{V}$ satisfy

$$
\nabla^{2}\left(\nabla^{2}-\frac{1}{v} \frac{\partial}{\partial t}\right) \mathbf{V}=0
$$

and

$$
\nabla \cdot \mathbf{V}=0
$$

Then $\mathbf{V}$ will represent the velocity of a possible unsteady Stokes flow if and only if the vorticity $\nabla \times \mathbf{V}$ satisfies the equation

$$
\left(\nabla^{2}-\frac{1}{v} \frac{\partial}{\partial t}\right)(\nabla \times \mathbf{V})=0 .
$$

Proof. Suppose $\mathbf{V}$ is the velocity of a possible unsteady Stokes flow in the absence of body forces. Then consider the Stokes equations

$$
\mu\left(\nabla^{2}-\frac{1}{v} \frac{\partial}{\partial t}\right) \mathbf{V}=\nabla p
$$


We have already seen that on operating curl on both sides of the above equation, we find that

$$
\left(\nabla^{2}-\frac{1}{v} \frac{\partial}{\partial t}\right)(\nabla \times \mathbf{V})=0
$$

Conversely, let $\nabla \times \mathbf{V}$ satisfy eq. (53). Then we show that $\mathbf{V}$ represents a possible unsteady, Stokes flow. As $\mathbf{V}$ is divergence-free, following the result given in [1], we can express $\mathbf{V}$ as

$$
\mathbf{V}=\nabla \times \nabla \times(\mathbf{r} A)+\nabla \times(\mathbf{r} B),
$$

where $A$ and $B$ satisfy the equations

$$
\begin{aligned}
& L A=-\mathbf{r} \cdot \mathbf{V}, \\
& L B=-\mathbf{r} \cdot(\nabla \times \mathbf{V}) .
\end{aligned}
$$

Further, as $\mathbf{V}$ and $\nabla \times \mathbf{V}$ satisfy eqs (4) and (53) respectively, the functions $A$ and $B$ will satisfy equations $\nabla^{2}\left(\nabla^{2}-\frac{1}{v} \frac{\partial}{\partial t}\right) A=0$ and $\left(\nabla^{2}-\frac{1}{v} \frac{\partial}{\partial t}\right) B=0$ respectively. Then $\mathbf{V}$ given in (24) and

$$
p=p_{0}+\mu \frac{\partial}{\partial r}\left[r\left(\nabla^{2}-\frac{1}{v} \frac{\partial}{\partial t}\right) A\right],
$$

is a solution of eqs (1) and (2). Hence $\mathbf{V}$ represents the velocity of a possible unsteady Stokes flow in the absence of any body forces.

In fact, as any solution of (4) can be expressed as $\mathbf{V}=\mathbf{V}_{1}+\mathbf{V}_{2}$ where

$$
\nabla^{2} \mathbf{V}_{1}=0, \quad\left(\nabla^{2}-\frac{1}{v} \frac{\partial}{\partial t}\right) \mathbf{V}_{2}=0
$$

we observe that from (53), the necessary and sufficient condition stated above reduces to the condition that

$$
-\frac{1}{v} \frac{\partial}{\partial t}\left(\nabla \times \mathbf{V}_{1}\right)=0
$$

Now we show that every harmonic $p$ represents the pressure in an unsteady Stokes flow in the absence of any body forces.

For a given $p$, we can choose a function $\phi$ and a function $A_{p}$ as in eqs (37) and (38), where

$$
\phi+r \frac{\partial \phi}{\partial r}=p
$$

and

$$
\mu\left(\nabla^{2}-\frac{1}{v} \frac{\partial}{\partial t}\right) A_{p}=\phi,
$$

where

$$
\nabla^{2}\left(\nabla^{2}-\frac{1}{v} \frac{\partial}{\partial t}\right) A_{p}=0
$$


Then we can show that

$$
\begin{aligned}
& \mathbf{V}_{1}=\nabla \times \nabla \times\left(\mathbf{r} A_{p}\right), \\
& p=\mu \frac{\partial}{\partial r}\left[r\left(\nabla^{2}-\frac{1}{v} \frac{\partial}{\partial t}\right) A_{p}\right],
\end{aligned}
$$

is a possible unsteady Stokes flow in the absence of any body forces.

Observe that for a given velocity field $\mathbf{V}$, the pressure $p$ is determined uniquely up to a constant. For a given harmonic $p,\left(\mathbf{V}_{1}, p\right)$ is a particular solution of Stokes equations and $(\mathbf{V}, p)$ is a solution of eqs (1) and (2) if $\left(\mathbf{V}-\mathbf{V}_{1}\right)$ satisfies

$$
\left(\nabla^{2}-\frac{1}{v} \frac{\partial}{\partial t}\right)\left(\mathbf{V}-\mathbf{V}_{1}\right)=0
$$

\section{Conclusions}

Some solutions of homogeneous and non-homogeneous, unsteady Stokes equations have been discussed and their completeness has been established. In the case of homogeneous equations, we found a solution of the Naghdi-Hsu [3] type which is shown to be complete. However, the solution that is derived in the more general case, i.e., in the case of nonhomogeneous equations in the presence of body forces, is more advantageous as it does not involve an integral and yet is in a closed form. It is also easy to employ this solution for boundary value problems as the boundary conditions can be expressed more easily using the scalar functions that appear in this solution. This fact has been observed in the steady case also [56]. In fact, this solution is also valid in an infinite domain which follows by employing the proof given in [4]. Lastly, a necessary and sufficient condition has been derived for a divergence-free vector field to be a solution of a possible unsteady Stokes flow in the absence of body forces.

\section{Appendix}

Suppose

$$
\nabla^{2}\left(\nabla^{2}-\frac{1}{v} \frac{\partial}{\partial t}\right) \psi=0
$$

then

$$
\psi=\psi_{1}+\psi_{2}
$$

where

$$
\begin{aligned}
& \nabla^{2} \psi_{1}=0 \\
& \left(\nabla^{2}-\frac{1}{v} \frac{\partial}{\partial t}\right) \psi_{2}=0
\end{aligned}
$$

Proof. Let

$$
\psi^{\prime}=\left(\nabla^{2}-\frac{1}{v} \frac{\partial}{\partial t}\right) \psi
$$


This implies

$$
\nabla^{2} \psi^{\prime}=0
$$

Define

$$
\psi_{2}=\psi+\int_{0}^{t} v \psi^{\prime}(x, y, z, s) \mathrm{d} s
$$

Then

$$
\begin{aligned}
\left(\nabla^{2}-\frac{1}{v} \frac{\partial}{\partial t}\right) \psi_{2} & =\left(\nabla^{2}-\frac{1}{v} \frac{\partial}{\partial t}\right) \psi+\left(\nabla^{2}-\frac{1}{v} \frac{\partial}{\partial t}\right) \int_{0}^{t} v \psi^{\prime}(x, y, z, s) \mathrm{d} s \\
& =\psi^{\prime}-\psi^{\prime}, \quad \text { using (A1) and (A2) } \\
& =0
\end{aligned}
$$

Let

$$
\psi_{1}=-\int_{0}^{t} v \psi^{\prime}(x, y, z, s) \mathrm{d} s
$$

Hence

$$
\psi=\psi_{1}+\psi_{2}
$$

where

$$
\begin{aligned}
& \nabla^{2} \psi_{1}=0 \\
& \left(\nabla^{2}-\frac{1}{v} \frac{\partial}{\partial t}\right) \psi_{2}=0
\end{aligned}
$$

\section{References}

[1] Chadwick P and Trowbridge E A, Elastic wave fields generated by scalar wave functions, Proc. Camb. Phil. Soc. 63 (1967) 1177-1187

[2] McBain G D, Convection in a horizontally heated sphere, J. Fluid Mech. 438 (2001) $1-10$

[3] Naghdi P M and Hsu C S, On a representation of displacements in linear elasticity in terms of three stress functions, J. Math. Mech. 10 (1961) 233-245

[4] Padmavathi B S and Amaranath T, A note on decomposition of solenoidal fields, Appl. Math. Lett. 15 (2002) 803-805

[5] Padmavathi B S, Rajasekhar G P and Amaranath T, A note on complete general solutions of Stokes equations, Q. J. Mech. Appl. Math. 51(3) (1998) 383-388

[6] Palaniappan D, Nigam S D, Amaranath T and Usha R, Lamb's solution of Stokes equations: a sphere theorem, Q. J. Mech. Appl. Math. 45(1) (1992) 47-56 